PROCEEDINGS OF THE

AMERICAN MATHEMATICAL SOCIETY

Volume 134, Number 12, December 2006, Pages 3485-3486

S 0002-9939(06)08438-3

Article electronically published on June 19, 2006

\title{
PERIODIC GROUPS WHOSE SIMPLE MODULES HAVE FINITE CENTRAL ENDOMORPHISM DIMENSION
}

\author{
ROBERT L. SNIDER
}

(Communicated by Martin Lorenz)

\begin{abstract}
Theorem. If $k$ is an uncountable field and $G$ is a periodic group with no elements of order the characteristic of $k$ and if all simple $k[G]$ modules have finite central endomorphism dimension, then $G$ has an abelian subgroup of finite index.
\end{abstract}

Let $k$ be a field and let $G$ be a group. There has been considerable interest in classifying those group rings $k[G]$ whose simple modules are finite dimensional in some sense. Three different notions of finite dimension have been studied. They are finite dimensional over $k$, finite dimensional over the division ring of endomorphisms, or finite dimensional over the center of the division ring of endomorphisms. A simple module satisfying this last condition is said to have finite central endomorphism dimension. The problem started with Philip Hall who proved that if $k$ is algebraic over a finite field and $G$ is a finitely generated nilpotent group, then each simple module is finite dimensional over $k$. On the other hand, if $k$ is any other field and $G$ is still finitely generated nilpotent, then all simple $k[G]$ modules having finite $k$ dimension implies the group is abelian-by-finite [1. This was extended to polycyclic groups by Roseblade 5. Solvable groups and locally finite groups have been studied 2, 8, 9, 7, 10, 11, 12. An attack on the general case was initiated by Passman and Temple [6. But one case that has remained untouched is the case of a periodic nonlocally finite group.

These conditions can all be stated in terms of the primitive images. Simple modules finite dimensional over the endomorphism ring is are equivalent to the primitive images being simple Artin rings. Finite dimensional over $k$ is equivalent to primitive images being finite dimensional over $k$ and finite central endomorphism dimension is equivalent to primitive images being finite dimensional over their centers.

Theorem 1. If $k$ is an uncountable field and $G$ is a periodic group with no elements of order the characteristic of $k$ and if all simple $k[G]$ modules have finite central endomorphism dimension, then $G$ has an abelian subgroup of finite index.

Proof. We can assume that $G$ is finitely generated [6, Corollary 1]. We want to show that $G$ is actually finite. The hypothesis implies that a primitive factor ring is a matrix ring over a division algebra finite dimensional over its center. The finite dimensionality implies that the image of $G$ is a linear group, and hence the image is

Received by the editors June 16, 2005 and, in revised form, July 19, 2005.

2000 Mathematics Subject Classification. Primary 16S34, $20 \mathrm{C07.}$

Key words and phrases. Group rings, periodic groups.

(C)2006 American Mathematical Society Reverts to public domain 28 years from publication 
finite by Burnside's theorem. Therefore each simple module is a actually a simple module for some finite image of $G$. Since $G$ is finitely generated, there are only countably many subgroups of finite index and hence only countably many finite images. Since each finite image has only finitely many simple modules, $k[G]$ has only countably many simple modules and only countably many primitive ideals. Let $a \in k[G]$. In each primitive image, $a$ has only finitely many eigenvalues. Hence for $\lambda \in k$ outside a countable set $S$, each $a-\lambda$ is invertible in each primitive image and $(a-\lambda) k[G]$ is a right ideal. If it is proper, then there is a maximal right ideal $\rho$ containing $(a-\lambda) k[G]$. But $\lambda$ is not an eigenvalue for $a$ when acting on the simple module $k[G] / \rho$. Therefore $a-\lambda$ is right invertible. Since groups rings have an involution, the condition is left-right symmetric. Hence outside a countable set, $a-\lambda$ is left invertible and hence invertible. Now $a$ is algebraic over $k$. If not, then the $(a-\lambda)^{-1}$ are all linearly independent for all $\lambda \notin S$. But $G$ is countable, whence $k[G]$ has countable dimension. Therefore $k[G]$ is an algebraic algebra. Now $k[G]$ is semiprimitive [4, p. 273]. The hypothesis implies that each primitive image satisfies a polynomial identity. A theorem of Kaplansky [3, Theorem 10.3] implies that $k[G]$ is locally finite dimensional. Therefore $G$ is locally finite, and hence $G$ is finite.

In the case where the field has characteristic 0 , one can use a much easier theorem of Herstein [4, p. 53] instead of the Kaplansky theorem.

\section{REFERENCES}

[1] P. Hall, On the finiteness of certain soluble groups, Proc. London Math. Soc. (3) 9(1959), 595-622. MR.0110750 (22:1618)

[2] B. Hartley, Locally finite groups whose irreducible modules are finite dimensional, Rocky Mountain J. Math. 13(1983), 255-263. MR0702822 (85e:20008)

[3] I. Kaplansky, The structure of certain operator algebras, Trans. Amer. Math. Soc. 70(1951), 219-255. MR0042066 (13:48a)

[4] D. S. Passman, The algebraic structure of group rings, Wiley-Interscience [John Wiley \& Sons], 1977. MR0470211 (81d:16001)

[5] J. E. Roseblade, Group rings of polycyclic groups, J. Pure Appl. Algebra 3(1973), 307-328. MR.0332944 (48:11269)

[6] D. S. Passman and W. V. Temple, Groups with all irreducible modules of finite degree, Algebra (Moscow, 1998), 263-279. MR1754674 (2001f:20017)

[7] B. A. F. Wehrfritz, Groups whose irreducible representations have finite degree, Math. Proc. Cambridge Philos. Soc. 90(1981), 411-421. MR0628826 (83a:20011)

[8] R. L. Snider, Solvable groups whose irreducible modules are finite dimensional, Comm. Algebra 10(1982), 1477-1485. MR0662712 (84a:20010)

[9] R. L. Snider, Group rings with finite endomorphism dimension, Arch. Math. (Basel) 41(1983). MR0721053 (85i:16015)

[10] B. A. F. Wehrfritz, Groups whose irreducible representations have finite degree, II, Proc. Edinburgh Math. Soc. (2) 25(1981), 237-243. MR0678547 (84b:20042a)

[11] B. A. F. Wehrfritz, Groups whose irreducible representations have finite degree, III, Math. Proc. Cambridge Philos. Soc. 91(1982), 397-406. MR0654085 (84b:20042b)

[12] B. A. F. Wehrfritz, Group rings with finite central endomorphism dimension, Glasgow Math. J. 24(1983), 169-176. MR0706146 (85c:20007)

Department of Mathematics, Virginia Polytechnic Institute and State University, BLACKSBURG, ViRGINIA 24061-0123

E-mail address: snider@math.vt.edu 